\title{
Efficacy of S-1 after Pemetrexed in Patients with Non-small Cell Carcinoma: A Retrospective Multi- institutional Analysis
}

Shinnosuke Takemoto ( $\nabla$ shinnosuke-takemoto@umin.ac.jp )

Nagasaki University Hospital

Kazumasa Akagi

Nagasaki Harbor Medical Center

Sawana Ono

Nagasaki Prefecture Shimabara Hospital

Hiromi Tomono

Sasebo City General Hospital

Noritaka Honda

Nagasaki University Hospital

Takayuki Suyama

Nagasaki University Hospital

Yasuhiro Umeyama

Nagasaki University Hospital

Yosuke Dotsu

Nagasaki University Hospital

Hirokazu Taniguchi

Nagasaki University Hospital

Daiki Ogawara

Sasebo City General Hospital

Hiroaki Senju

Nagasaki University Hospital

Hiroshi Gyotoku

Nagasaki University Hospital

Nanae Sugasaki

Nagasaki Prefecture Shimabara Hospital

Hiroyuki Yamaguchi

Nagasaki University Hospital

Katsumi Nakatomi

Ureshino Medical Center

Minoru Fukuda 
Nagasaki University Hospital

\section{Hiroshi Mukae}

Nagasaki University Hospital

\section{Research Article}

Keywords: Pemetrexed, S-1, lung cancer, cross-resistance

Posted Date: December 28th, 2020

DOl: https://doi.org/10.21203/rs.3.rs-122720/v1

License: (1) This work is licensed under a Creative Commons Attribution 4.0 International License. Read Full License 


\section{Abstract}

Back ground: This study was designed to evaluate the treatment effect of S-1 following PEM-containing treatment.

Methods: This retrospective study included patients with advanced (c-stage III or IV, UICC 7th) or recurrent NSCLC who received S-1 monotherapy following the failure of previous PEM-containing chemotherapy at 6 hospitals in Japan.

Primary endpoint: Overall response rate (ORR). Secondary endpoint: Disease control rate (DCR), time to treatment failure (TTF), progression-free survival (PFS), and overall survival (OS).

Results: A total of 53 NSCLC patients met the criteria. Forty-six patients had adenocarcinoma (88.7\%) and no patients had squamous cell carcinoma. Thirty-one patients $(58.5 \%)$ received the standard S-1 regimen and 18 patients $(34.0 \%)$ received the modified S-1 regimen. ORR was 1.9\% (95\% confidential interval $(\mathrm{Cl})$ : 0.00-10.1\%). Median TTF, PFS, and OS were 65 days, 84 days, and 385 days, respectively.

Conclusion: Although there were several limitations in this study, the ORR of S-1 after PEM in patients with non-SQ NSCLC was low compared to the historical control. It might be one of the choices to avoid S1 treatment in PEM-treated patients who need tumor shrinkage.

\section{Introduction}

S-1 and pemetrexed (PEM) are key treatments for non-small cell lung cancer (NSCLC). PEM and cisplatin (CDDP), showed superior overall survival (OS) compared with gemcitabine and CDDP in treating nonsquamous (non-Sq) NSCLC patients, and PEM and platinum treatment is usually used for this population(1). S-1 monotherapy showed non-inferior OS compared with docetaxel in treated NSCLC patients, and is also used for NSCLC as a standard therapy after first line treatment(2). However, the mechanism of anticancer activity of S-1 and PEM is similar. For example, both S-1 and PEM target thymidylate synthase (TS)(3). Moreover, cross-resistance between S-1 and PEM is of concern. Some preclinical studies indicated that elevation of TS expression after PEM treatment may be one of the causes of cross-resistance between S-1 and $\operatorname{PEM}(4,5)$. In addition, TS expression level is associated with response to S-1 in NSCLC in a clinical setting(6). Resistance to PEM may indicate resistance to S-1. Unfortunately, studies about the treatment effect of S-1 after PEM in the clinical setting are limited.

\section{Aim Of This Study}

To evaluate the treatment effect of S-1 after PEM containing treatment.

\section{Patients And Methods}

\section{Selection of Patients}


This retrospective study included patients with advanced (c-stage III or IV, UICC 7th ) or recurrent NSCLC who received S-1 monotherapy following the failure of previous PEM containing chemotherapy at Nagasaki University Hospital, Nagasaki Harbor Medical Center, Sasebo City General Hospital, Nagasaki Medical Center, Shimabara Hospital, or Ureshino Medical Center between April 2012 and March 2017.

The full analysis set (FAS) included patients who 1) were administered S-1 for more than 15 days, 2) previously underwent three or less treatments prior to $S-1$, and 4) had target lesions. The medical records of eligible patients were reviewed retrospectively.

\section{Data collection}

The following items about the patients were collected from medical records: age, sex, pathology, smoking status, main medical histories, main comorbidities, epidermal growth factor receptor (EGFR) mutation status, anaplastic lymphoma kinase (ALK) fusion gene, clinical stage (UICC 7th ), Eastern Cooperative Oncology Group (ECOG) performance status (PS) at the date of S-1 administration, date of S-1 or PEM administration, medication method of $S-1$, number of treatment cycles, date of disease progression, date of final administration, reason for cessation of treatment, survival information, date of last follow-up, and number of treatments prior to S-1.

\section{Statistical analysis}

The present study is a multi-institutional retrospective observational study including six institutes. Primary endpoint is overall response rate (ORR), which includes partial response (PR) and complete response (CR). Secondary endpoints are disease control rate (DCR), time to treatment failure (TTF), progression-free survival (PFS), and OS. Tomita et al. previously reported that the ORR of S-1 was 9\%(7). This study was selected as a historical control because this cohort was similar with the present study (the efficacy of S-1 was evaluated retrospectively). On the other hand, PEM was not administered before S-1 in most cases in this cohort because the pharmaceutical approval of PEM occurred in 2009 in Japan (Therapy period of S-1 in the cohort was between March 2004 and October 2010 in historical control). In this present study, expected ORR was set to $9 \%$ if there was no cross-resistance between PEM and S-1 and an unacceptable ORR due to cross-resistance was set to $4 \%$. The DCR, median TTF, PFS, and OS were also compared with the historical control. DCR included stable disease (SD), PR and CR. TTF was calculated from the date of the first day of S-1 monotherapy and the date of discontinuation of S-1. PFS was calculated using the date of the start of S-1 monotherapy and the date of disease progression, and OS was calculated using the date of the start of S-1 monotherapy and the date of mortality from any cause or the last follow-up. The Kaplan-Meier method was used to calculate PFS and OS. Tumor responses were assessed according to the Response Evaluation Criteria in Solid Tumors (RECIST) ver.1.1(8).

\section{Results}

The method of patient selection is shown in Fig. 1. A total of 53 NSCLC patients met the selection criteria, and this population was defined as the FAS. 
The patient characteristics are shown in Table 1. Of the total 53 patients, 26 patients (49.0\%) were < 70 years of age. Age, PS, smoking history, staging, and EGFR gene mutation status were similar with the historical control(7). There were no patients with ALK fusion gene. Forty-six patients had adenocarcinoma (88.7\%) and no patients had squamous cell carcinoma. Regarding treatment delivery, 31 patients (58.5\%) received the standard S-1 regimen (4 weeks administration followed by 2 weeks of rest, every 6 weeks) and 18 patients (34.0\%) received the modified S-1 regimen (2 weeks administration followed by 1 week of rest, every 3 weeks) for the first S- 1 cycle. Twenty-four patients received 5 or more PEM rounds of administration. The median period between last PEM administration and first S-1 administration was 118 days (range: 11-625 days). No immune check point inhibitors (ICls) were administered between PEM and S-1.

The treatment efficacy of S-1 is shown in Table 2. ORR was $1.9 \%$ (95\% confidential interval [CI]: 0.00 $10.1 \%$ ) and DCR was $41.5 \%$ (95\% Cl: 28.1-55.9\%). Median TTF, PFS and OS were Collating preplanned criteria, the treatment effect of S-1 after PEM, might be less than that of the no prior PEM treatment population. Moreover, in the historical control, especially the adenocarcinoma subset, ORR was $15.8 \%$ (95\% Cl: $3.3-39.8 \%)$ and DCR was $57.8 \%$ (95\% Cl: 33.5-79.7\%)(7).

Median TTF, PFS, and OS in this study were 65 days, 84 days, and 385 days, respectively (Fig. 2). In the adenocarcinoma subset of historical control, median PFS and OS were 4.2 months and 15.7 months, respectively (TTF was not shown). Compared with historical control, PFS and OS in this study tended to be worse.

To search for the predictive factor of S-1 effect after PEM containing treatment, differential analysis was used about two factors. One was the number of PEM administration and the other was the period between last PEM administration and first S-1 administration. ORR was too low to analyze, TTF and PFS were used as a surrogate of efficacy. Between-group differences in TTF and PFS were assessed using the stratified log-rank test. Both of two factors could not predict the efficacy of S-1 after PEM treatment (Fig. 3, Fig. 4). However, the longer period between last PEM and first S-1 group tended to longer PFS and TTF.

\section{Discussion}

S-1 is the standard treatment for previously treated NSCLC in the clinical setting(9). However, ORR of S-1 after PEM in the present study seemed to have less anti-tumor effect than historical control.

DCR, PFS and OS also showed similar tendencies, the difference was modest. Banqu et al. reported a relationship between TS expression levels and the ability to acquire resistance to antifolates using 5 PEM resistant cell lines(5). In addition, Takeda et al. reported immunohistochemical expression levels of TS and the response to treatment with S-1 in NSCLC; comparing S1 plus carboplatin with paclitaxel plus carboplatin, PFS in the low TS group tended to be longer than in the high TS group in SC patients, and there was no difference among the PC group(6). It was presumed that one of the mechanisms of crossresistance between PEM and S-1 was reduction of TS expression due to prior PEM treatment. To compare 
this study, previous reports about S-1 monotherapy were checked up (Table 3)(7, 9-14). Interestingly, in two studies about efficacy of S-1 whose registration period was prior to 2009, S-1 showed higher ORR in adenocarcinoma or non-SQ than in squamous cell carcinoma $(11,12)$. PEM was probably not administered to the analyzed populations because the efficacy of PEM was not improved in clinical trials at the time. After 2009, PEM containing tratment was usually used for non-Sq NSCLC. In 2016, randomized phase III trial comparing S-1 with docetaxel (DTX) in patients with non-Sq NSCLC patients previously treated with platinum-based chemotherapy was reported. Subset analysis of this study suggested that PFS of S-1 was inferior to PFS of DTX in adenocarcinoma(9). In this population, many non-Sq NSCLC (mainly adenocarcinoma) patients received PEM treatment because the registration period of this study was between July 2010 and June 2014. These evidences reinforce that prior PEM treatment weaken anti-tumor effects of S-1 and support the presence of cross-resistance between PEM and S-1.

No ICls were administered between PEM and S-1 in this study. Grigg et al. reported that some chemotherapies may act through immune-mediated mechanisms and chemotherapy response rates may be higher when administered after ICIs(15). Furthermore, S-1 after PEM and ICI treatment might show higher ORR.

Exploratory analysis about predictive factor of S-1 after PEM suggested the longer period between last PEM administration and first S-1 administration. It might be one of the choices to avoid S-1 treatment immediately after PEM.

There are several limitations in this study. Firstly, there was no control data comparing with the population in this study. This may have impacted our findings; for example, there were more PS and smoking patients in our study compared with the historical control, and patient characteristics in this study were slightly different from the historical control (e.g. containing $11.3 \%$ non-adeno-non-squamous NSCLC cases). However, there were only a few patients administered with S-1 without prior PEM. Secondly, the sample size in this study was small and our study findings may be inadvertent. Thirdly, there was no diagnostic radiology central review in this study. Fourthly, more modified regimen was used in this study than historical control (41.5\% vs $9.3 \%)(7)$. The difference of treatment schedule might affect the efficacy.

\section{Conclusion}

The efficacy of S-1 after PEM in patients with NSCLC showed low ORR compared with the historical control. It might be one of the choices to avoid S-1 treatment in PEM-treated patients who need more possibility of tumor shrinkage.

\section{Declarations}

\section{Ethics approval and consent to participate:}


The study protocol was reviewed and approved by the Institution Review Board of the Nagasaki Medical Center, Nagasaki University Hospital, Ureshino Medical Center, Sasebo City General Hospital, Nagasaki Harbor Medical Center, and Nagasaki Prefecture Shimabara Hospital (UMIN ID:000033374). Informed consent and consent for publication were obtained in the form of opt-out on the web site. The study was performed in accordance with the Declaration of Helsinki.

\section{Funding:}

Funding were not applicable.

\section{Competing interest:}

Competing interests were not applicable.

\section{Availability of data and materials:}

All data generated or analyzed during this study are included in this published article and its supplementary information files.

\section{Author's contributions:}

A was Shinnosuke Takemoto, B was Kazumasa Akagi, C was Sawana Ono, D was Hiromi Tomono, E was Noritaka Honda, F was Takayuki Suyama, G was Yasuhiro Umeyama, H was Yosuke Dotsu, I was Hirokazu Taniguchi, J was Daiki Ogawara, K was Hiroaki Senju, L was Hiroshi Gyotoku, M was Nanae Sugasaki, N was Hiroyuki Yamaguchi, O was Katsumi Nakatomi, P was Minoru Fukuda, Q was Hiroshi Mukae. A collected datasets about Nagasaki Medical Center. B collected datasets about Nagasaki Harbor Medical Center. C and M collected datasets about Nagasaki Prefecture Shimabara Hospital. D and J collected datasets about Sasebo City General Hospital. E, F, G, H, I, K, L, N, P and Q collected datasets about Nagasaki University Hospital. O collected datasets about Ureshino Medical Center.. All authors read and approved the final manuscript.

\section{Acknowledgements:}

This study was in part supported by Shuntaro Sato, Clinical Research Center, Nagasaki University Hospital, Nagasaki, Japan.

\section{References}


1. Scagliotti GV, Parikh P, Pawel J von, Biesma B, Vansteenkiste J, Manegold C, et al. Phase III Study Comparing Cisplatin Plus Gemcitabine With Cisplatin Plus Pemetrexed in Chemotherapy-Naive Patients With Advanced-Stage Non-Small-Cell Lung Cancer. J Clin Oncol. 2008;26(21):3543-51.

2. Nokihara H, Lu S, Mok TSK, Nakagawa K, Yamamoto N, Shi YK, et al. Randomized controlled trial of S-1 versus docetaxel in patients with non-small-cell lung cancer previously treated with platinumbased chemotherapy (East Asia S-1 Trial in Lung Cancer). Ann Oncol Official J European Soc Medical Oncol. 2017;28(11):2698-706.

3. Maring JG, Groen HJM, Wachters FM, Uges DRA, Vries EGE de. Genetic factors influencing Pyrimidine-antagonist chemotherapy. Pharmacogenomics J. 2005;5(4):226-43.

4. YANG M, FAN W-F, PU X-L, LIU F-Y, MENG L-J, WANG J. Significance of thymidylate synthase expression for resistance to pemetrexed in pulmonary adenocarcinoma. Oncol Lett. 2014;7(1):22732.

5. Buqué A, Aresti U, Calvo B, Muhialdin JS, Muñoz A, Carrera S, et al. Thymidylate synthase expression determines pemetrexed targets and resistance development in tumour cells. Plos One. 2013;8(5):e63338.

6. Takeda M, Okamoto I, Hirabayashi N, Kitano M, Nakagawa K. Thymidylate synthase and dihydropyrimidine dehydrogenase expression levels are associated with response to S-1 plus carboplatin in advanced non-small cell lung cancer. Lung Cancer Amsterdam Neth. 2010;73(1):1039.

7. TOMITA Y, OGURI T, TAKAKUWA O, NAKAO M, KUNII E, UEMURA T, et al. S-1 monotherapy for previously treated non-small cell lung cancer: A retrospective analysis by age and histopathological type. Oncol Lett. 2011;3(2):405-10.

8. Eisenhauer EA, Therasse P, Bogaerts J, Schwartz LH, Sargent D, Ford R, et al. New response evaluation criteria in solid tumours: Revised RECIST guideline (version 1.1). Eur J Cancer. 2009;45(2):228-47.

9. Nishio M, Mok TSK, Nakagawa K, Yamamoto N, Shi Y-K, Zhang L, et al. EAST-LC: Randomized controlled phase III trial of S-1 versus docetaxel in patients with non-small-cell lung cancer who had received a platinum-based treatment. Ann Oncol. 2016;27(suppl_6).

10. Shiroyama T, Komuta K, Imamura F, Hirashima T, Kijima T, Tachibana I, et al. Phase II study of S-1 monotherapy in platinum-refractory, advanced non-small cell lung cancer. Lung Cancer. 2011;74(1):85-8.

11. Govindan R, Morgensztern D, Kommor MD, Herbst RS, Schaefer P, Gandhi J, et al. Phase II Trial of S-1 as Second-Line Therapy in Patients with Advanced Non-small Cell Lung Cancer. J Thorac Oncol. 2011;6(4):790-5.

12. Group) S-1 CSG (Lung CW, Kawahara M, Furuse K, Segawa Y, Yoshimori K, Matsui K, et al. Phase II study of S-1, a novel oral fluorouracil, in advanced non-small-cell lung cancer. Brit J Cancer. 2001;85(7):939-43. 
13. Totani Y, Saito Y, Hayashi M, Tada T, Kohashi Y, Mieno Y, et al. A phase II study of S-1 monotherapy as second-line treatment for advanced non-small cell lung cancer. Cancer Chemoth Pharm. 2009;64(6):1181.

14. Kasai T, Nakamura Y, Fukuda M, Kitazaki T, Nagashima S, Takatani H, et al. A Phase II Study of S-1 for Previously Untreated Elderly Patients with Advanced Non-Small Cell Lung Cancer. Chemotherapy. 2016;61(2):93-8.

15. Grigg C, Reuland BD, Sacher AG, Yeh R, Rizvi NA, Shu CA. Clinical outcomes of patients with nonsmall cell lung cancer (NSCLC) receiving chemotherapy after immune checkpoint blockade. J Clin Oncol. 2017;(35):(suppl; abstr 9082).

\section{Tables}

Due to technical limitations, tables are only available as a download in the Supplemental Files section.

\section{Figures}

Fig. 1

NSCLC who received S-1 monotherapy following the failure of previous PEM containing chemotherapy patients $\mathrm{N}=84$

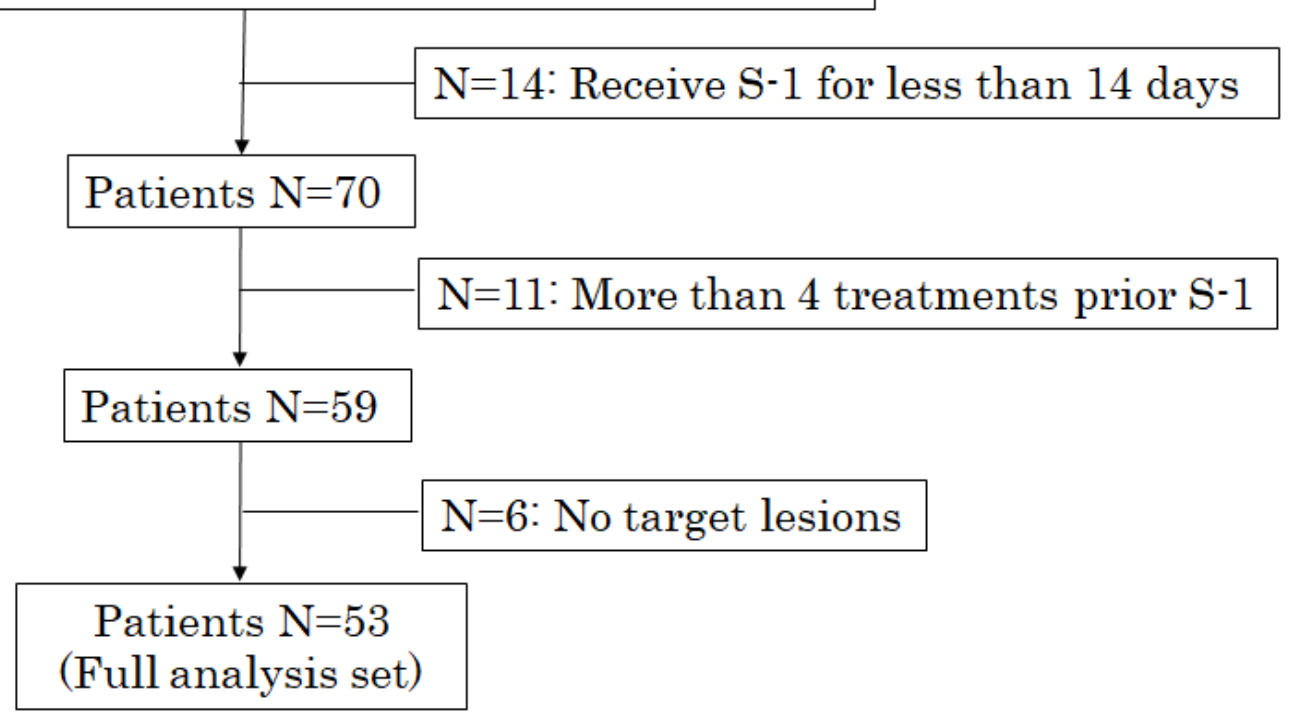

\section{Figure 1}

Scheme of full analysis set (FAS). 
Fig. 2
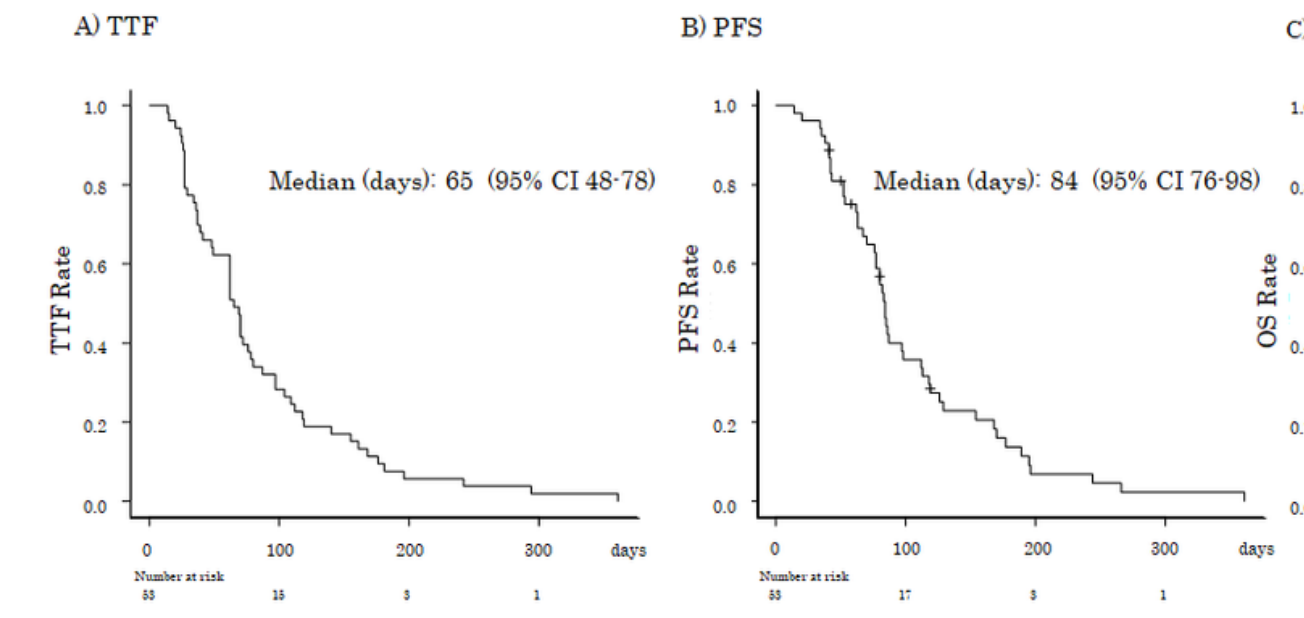

C) os

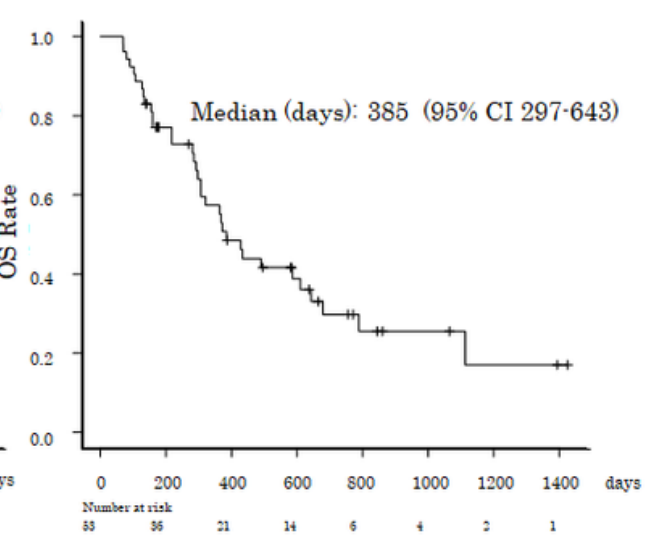

\section{Figure 2}

Kaplan-Meier curves of A) treatment failure (TTF), B) progression-free survival (PFS), and C) overall survival (OS) for the patients $(n=53)$ in the full analysis set.

\section{Fig. 3}

\section{A) $\mathrm{TTF}$}

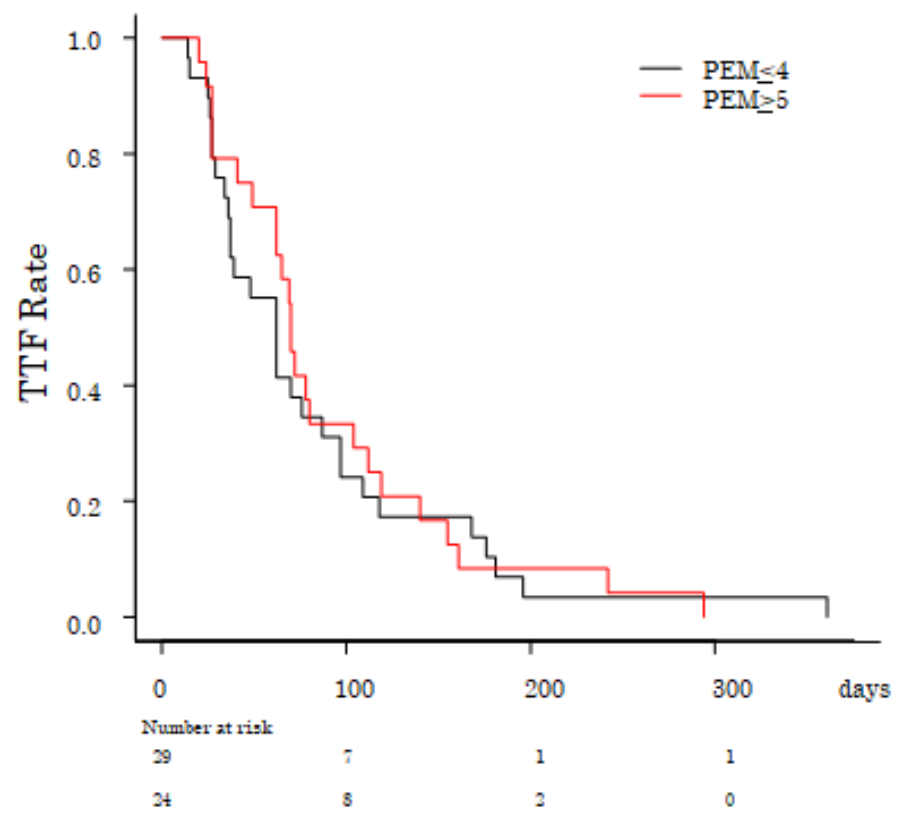

B) PFS

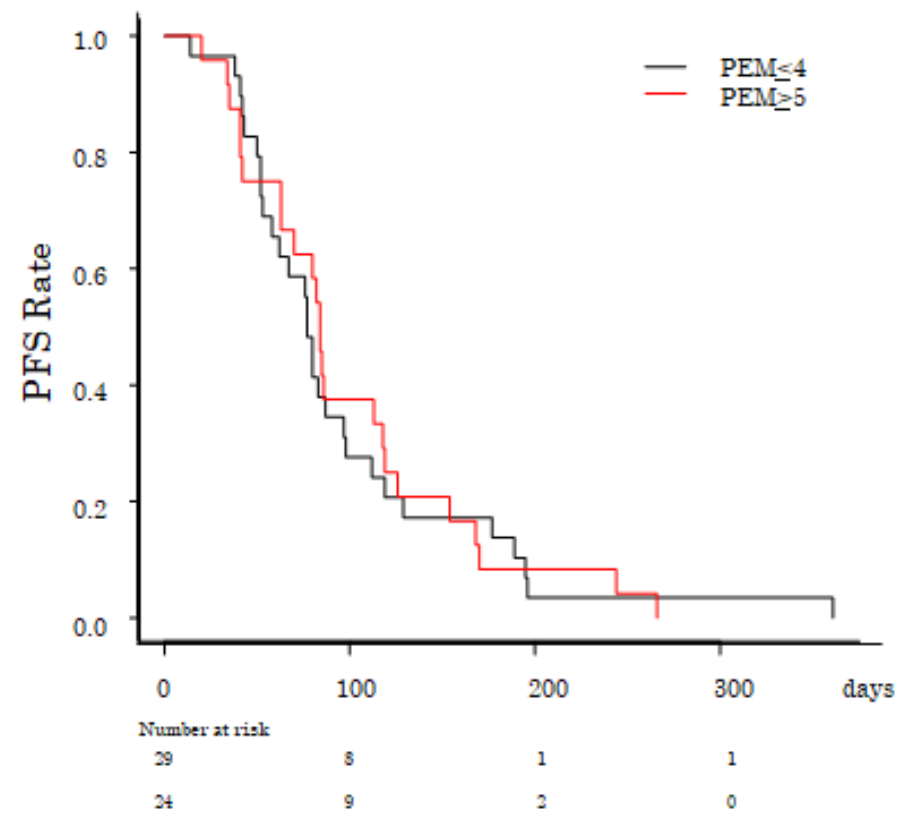

Figure 3 
Kaplan-Meier curves of A) TTF, B) PFS stratified by the number of PEM administration. Black line indicates subgroup PEM was administered 4 or less $(P E M<4)$ and Red line indicates 5 or more $(P E M>5)$. There was no significant defference between $\mathrm{PEM}<4$ group and $\mathrm{PEM}>5$ in median TTF ( 77 days vs 84 days, respectively; $\mathrm{P}=0.86$ ) and PFS (62 days vs 70 days, respectively; $\mathrm{P}=0.72$ ).

Fig. 4

A) TTF

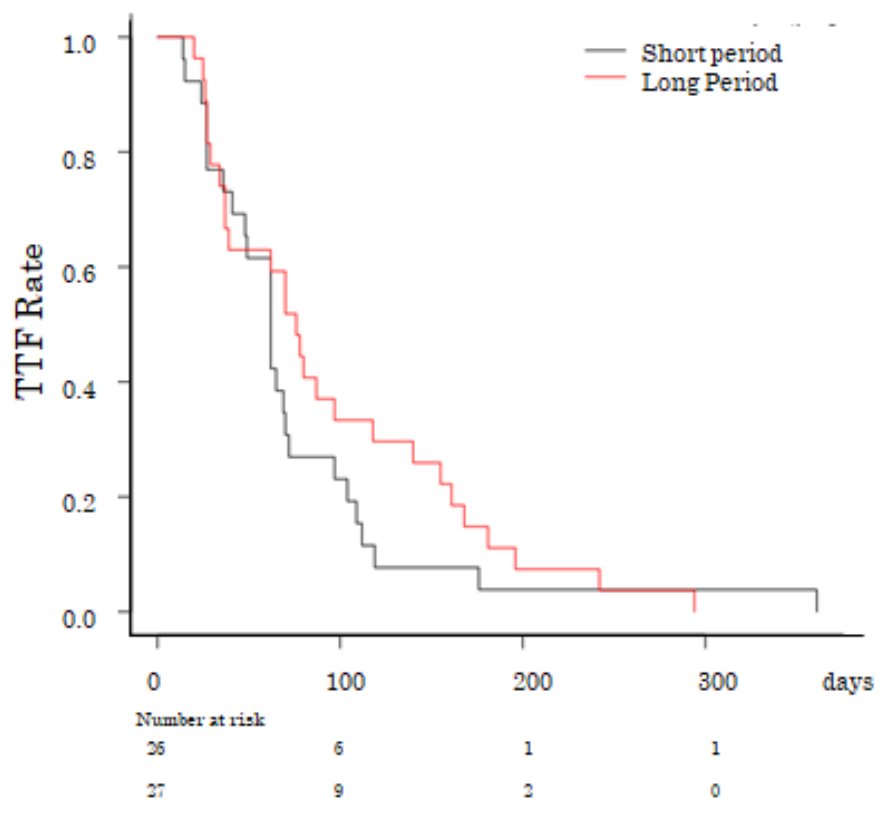

B) PFS

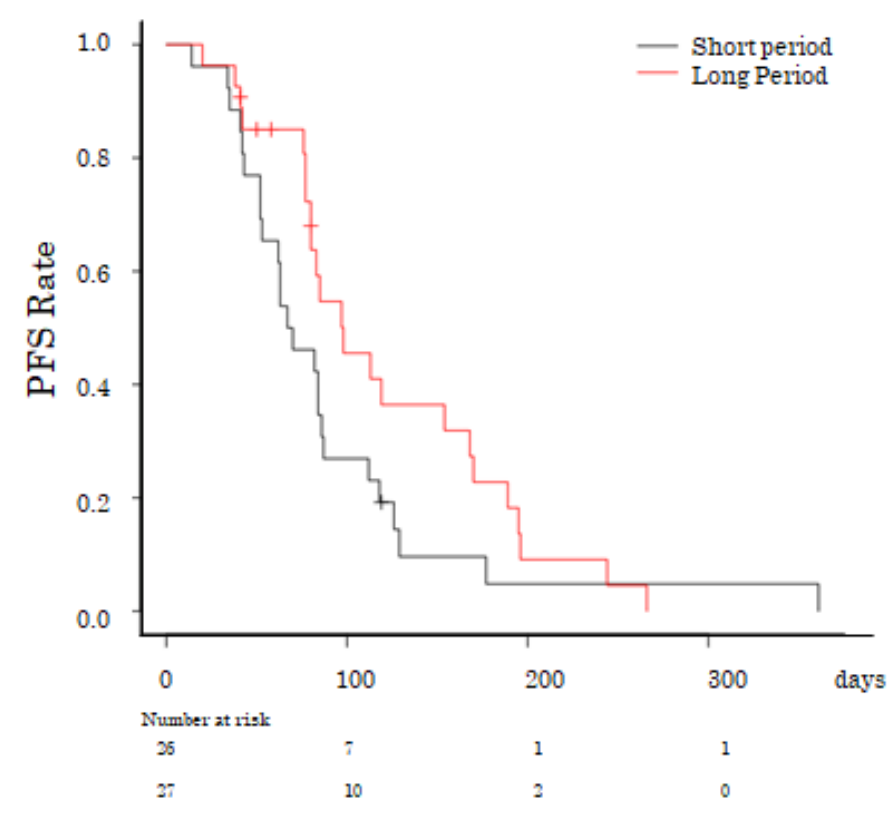

\section{Figure 4}

Kaplan-Meier curves of A) TTF, B) PFS stratified by the period between last PEM administration and first S-1 administration. Black line indicates subgroup the period was below the median (Short period) and Red line indicates above the median (Long period). There was no significant defference between Short period group and Long period group in median TTF ( 62 days vs 76 days, respectively; $P=0.29$ ) and PFS (68.5 days vs 98 days, respectively; $P=0.11$ ).

\section{Supplementary Files}

This is a list of supplementary files associated with this preprint. Click to download.

- table.pptx

- DataSet.xlsx 